\title{
Research on Cultural Tourism Product Development of "Impression Liu Sanjie" Based on the Law of Diminishing Marginal Utility
}

\author{
Heying $\mathrm{Li}^{1, *}$, Jinye Wang ${ }^{1}$ \\ ${ }^{1}$ Guilin University of Technology, Tourism and Landscape Architecture Department, Guilin, Guangxi541006, China
}

\begin{abstract}
According to the law of diminishing marginal utility, the marginal utility when consumers purchase a certain product shows a diminishing trend. As a special product, cultural tourism products, whether is the marginal utility produced during consumption also affected by the law of diminishing marginal utility. This paper takes "Impression Liu Sanjie" as the research object and uses a linear regression equation model to study the marginal utility of tourists "Impression Liu Sanjie" cultural tourism products. The results show that the marginal utility produced by tourists buying the cultural tourism products of "Impression Liu Sanjie" shows an obvious decreasing trend. The main reason is that the cultural tourism products of "Impression Liu Sanjie" lack innovation and strong brand characteristics, the overall scale is small, the positioning is not accurate, etc., affected by the competition of homogenized products in the surrounding area, and the return rate of tourists is low. Moreover, there is a gap between existing products and the development trend of high-end tourism, which cannot meet the needs of tourists for indepth experience and research tourism. Therefore, "Impression Liu Sanjie" needs to intensify innovation, fundamentally solve the problem of diminishing marginal utility, further stabilize the source of tourists and meet the needs of tourists for repeated consumption quality, and realize sustainable tourism development.
\end{abstract}

\section{Introduction}

Since the merger of the Ministry of Culture and the Ministry of Tourism into the Ministry of Culture and Tourism in 2018, the integration of culture and tourism and the development of cultural tourism have once again entered people's vision, becoming a new tourism trend and the focus of scholars. Through the literature search, the research on cultural tourism integration and cultural tourism mainly focuses on the traceability and development of local tourism culture ${ }^{[1]}$, the influence of traditional Chinese culture on tourism management ${ }^{[2]}$, the optimization design of cultural tourism products ${ }^{[3]}$ and culture Tourism development strategy ${ }^{[4-5]}$ etc. The law of diminishing marginal utility is put forward by cardinal utility theorists on the basis of psychology. When reaching a certain level, they believe that every time the use of a certain commodity increases, consumers' perception of it will decrease. The content of the law of diminishing marginal utility is that: In a certain period, under the condition that the consumption quantity of other commodities remains constant, as the consumption of a certain commodity made by consumers increases, the increase in utility that consumers obtain from each consumption unit where the commodity continuously increases is diminishing, in other words, the marginal utility is diminishing. ${ }^{[6]}$ The law of diminishing marginal utility is widely used in many research fields. In terms of tourism research, some scholars have studied the development of regional tourism and tourism products based on the law of diminishing marginal utility ${ }^{[7-9]}$, but there are relatively few studies on the development of cultural tourism products based on the law of diminishing marginal utility. The reason for the diminishing marginal utility in the operation of tourism products is not completely removed. Therefore, using the law of diminishing marginal utility to analyze the development of cultural tourism products is conducive to solving the problems in the development of cultural tourism, and is conducive to guiding the better integration of culture and tourism.

Impression Liu Sanjie is Zhang Yimou's fist "Landscape live performance" in China. "Landscape live performance" set up the theater on the waters of the $\mathrm{Li}$ River, with 12 mountains as the background and terraced fields as the auditorium, which vividly reflects a majestic style. Impression Liu Sanjie applied the largest environmental art lighting project, the unique smoke effect project and its hidden sound in China. It shows the five theme color series of "red, green, blue, gold, and silver". For a while, it has become one of the must-chosen items for visitors to Guilin, and it needs to be booked in advance. However, in recent years, due to the development of more homogeneous tourism products in the surrounding area, the attractiveness and operating efficiency of "Impression Liu Sanjie" have declined. In particular, the marginal utility of tourists buying "Impression Liu Sanjie" cultural tourism products has shown a clear decreasing trend, which seriously affected the development of "Impression Liu Sanjie". Therefore, this article applies the law of diminishing marginal utility, combines it with the theory 
of tourism product life cycle, takes "Impression Liu Sanjie" as the research object, analyzes the reasons for the diminishing marginal utility of tourists buying "Impression Liu Sanjie" cultural tourism products. This provides technical reference for solving the current problems of "Impression Liu Sanjie"'s declining competitiveness and low operating efficiency.

\section{2 "Impression Liu Sanjie"'s contribution to Yangshuo tourism and the marginal utility of cultural tourism products}

\section{1 "Impression Liu Sanjie"'s Contribution to Yangshuo Tourism}

Impression Liu Sanjie has achieved tremendous economic benefits since it was released. It has changed the situation in Yangshuo where tourists cannot stay overnight, brought a lot of direct employment opportunities, changed the life and production methods of local farmers, and promoted the rapid operation of the entire economic chain.

Table 1 Annual GDP and total tourism consumption in Yangshuo

\begin{tabular}{|c|c|c|}
\hline Year & $\begin{array}{c}\text { GDP (Billion/ } \\
\text { CNY) }\end{array}$ & $\begin{array}{c}\text { Total travel } \\
\text { consumption } \\
\text { (Billion/CNY) }\end{array}$ \\
\hline 2004 & 14.37 & 4.06 \\
\hline 2005 & 17.4 & 5.51 \\
\hline 2006 & 22.7 & 8.25 \\
\hline 2007 & 29.9 & 12.8 \\
\hline 2008 & 38.53 & 17.9 \\
\hline 2009 & 42.91 & 24.2 \\
\hline 2010 & 56.1 & 31.5 \\
\hline 2011 & 69.03 & 38.6 \\
\hline 2012 & 76.4 & 50.2 \\
\hline 2013 & 84.39 & 60.5 \\
\hline 2014 & 97.67 & 83.4 \\
\hline 2015 & 108.2 & 100.2 \\
\hline 2016 & 116.54 & 117.84 \\
\hline 2017 & 128.59 & 132.5 \\
\hline$D 20045$ & 6091 \\
\hline
\end{tabular}

Data source: Government Work Report and Guangxi Local Information Network

According to Table 1, the GDP and total tourism consumption of Yangshuo from 2004 to 2017 are plotted in Figure 1. The horizontal axis $\mathrm{X}$ represents the regional GDP and the vertical axis Y represents the total tourism revenue, and the corresponding trend line is drawn.

It can be seen from Figure 1 that the regional GDP of Yangshuo County and the total tourism consumption are roughly linearly related. And the linear regression method is used for analysis to construct an economic model of a linear regression equation. The model structure is:

$$
\mathrm{Y}=\mathrm{aX}+\mathrm{b}+\mathrm{e}
$$

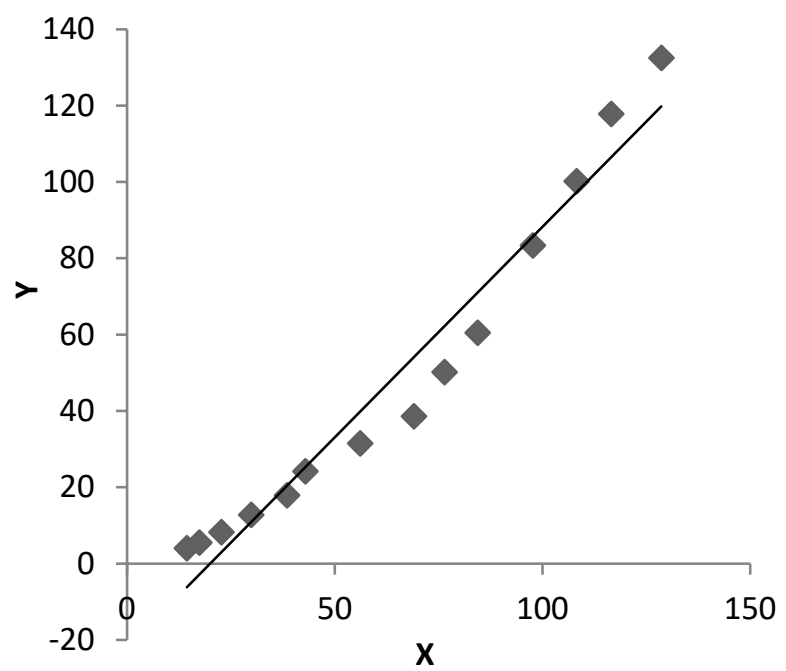

Figure 1 Regional GDP and tourism consumption of Yangshuo from 2004 to 2017

In formula (1), Y represents the gross production value of Yangshuo County, and $\mathrm{X}$ represents the total tourism consumption. Import the data in Table 1 into Excel and use regression analysis tools to calculate:

$$
\mathrm{Y}=1.103 \mathrm{X}-22.0193, r=0.97768
$$

The value range of $r$ is $[-1,1]$. The closer $|r|$ is to 1 , the closer the regression line is to each observation point, which is the higher fit of the regression line. When $|r| \geq 0.8$, the two variables can be regarded to be highly correlated. According to (2), $r=0.97768$, which is particularly close to 1. Therefore, the local GDP of Yangshuo County has a high degree of fitting with total tourism consumption. And it shows a highly positive correlation trend. Therefore, tourism is an important part of Yangshuo County, and the development of tourism has greatly promoted the development of the local economy. Impression Liu Sanjie is an important factor in attracting tourists in Yangshuo. From 2004 to 2013, Impression Liu Sanjie performed nearly 4,500 performances, received more than 10 million domestic and foreign audiences, including about 2 million overseas audiences (including Hong Kong, Macao and Taiwan). The total amount of taxes paid is close to 200 million Yuan.

\subsection{The Marginal Utility of "Impression Liu Sanjie" Cultural Tourism Products}

Assuming that "Impression Liu Sanjie" cultural tourism products have remained unchanged for more than ten years, the initial willingness to pay is set at $¥ 200$ for the convenience of calculation, and the marginal utility of currency is $\lambda=2$. It can be seen from Table 2, when the number of purchases of a tourist increases from 0 to 1 , the total utility increases from 0 to 400 , and its marginal utility, the increase in total utility, is equal to $400-0=400$; When the number of purchases of a tourist increases from 
1 to 2 , the total utility increases from 400 to 720 , and its marginal utility is $720-400=320$; by that analogy, when the number of consumption of a tourist increases to 6 , its total utility is 1200 , it remains unchanged when the number of purchases is 5, and the marginal utility here is 12000$1200=0$; when the number of purchases of a tourist is 7 , the total utility drops to 1180 , and the marginal utility is $1180-1200=-80$. The law of diminishing marginal utility is extremely obvious in tourism products. Normally, the scenic spots that a tourist has visited will not be visited once again.

Table 2 the utility table of Impression Liu Sanjie

\begin{tabular}{|c|c|c|c|}
\hline $\begin{array}{c}\text { Purchase } \\
\text { frequency }\end{array}$ & Total utility & $\begin{array}{c}\text { Marginal } \\
\text { utility }\end{array}$ & $\begin{array}{c}\text { Willing to } \\
\text { pay(China } \\
\text { Yuan) }\end{array}$ \\
\hline 0 & 0 & & \\
\hline 1 & 400 & 400 & 200 \\
\hline 2 & 720 & 320 & 160 \\
\hline 3 & 960 & 240 & 120 \\
\hline 4 & 1120 & 160 & 80 \\
\hline 5 & 1200 & 80 & 40 \\
\hline 6 & 1200 & 0 & 0 \\
\hline 7 & 1180 & -80 & \\
\hline
\end{tabular}

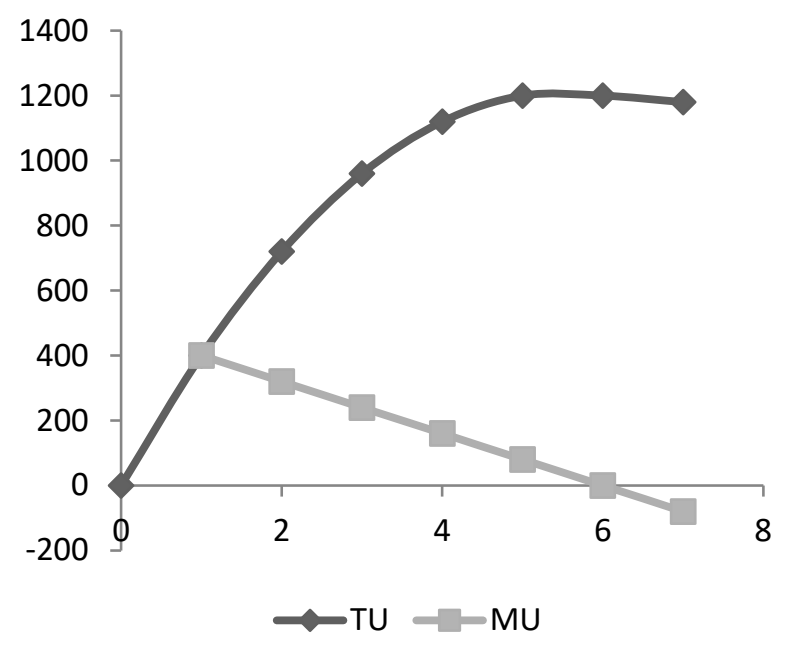

Figure 2 the utility curve of Impression Liu sanjie

As Figure 2 shows, the total utility curve and marginal utility curve of the tourist purchase impression Liu Sanjie are drawn from the values in Table 2. The horizontal axis is the number of times when a tourist purchases the impression of Liu Sanjie, and the vertical axis is the effective amount. It can be seen from the figure 2 that the MU curve slopes to the lower right, which just illustrates the law of diminishing marginal utility, indicating that every time a tourist increases the number of purchases of Impression Liu Sanjie, the marginal utility he obtains will decrease. When the marginal utility MU is greater than 0 , the total utility TU curve obtained by tourists shows an increasing trend, and the growth rate becomes slower; when the marginal utility $\mathrm{MU}$ is equal to 0 , the TU curve stops increasing; when the marginal utility MU is less than 0 , the TU curve begins to decline.

For tourists, if they have enough time, enough disposable income and desire to buy impression Liu Sanjie, he will buy it. If the tourist makes the first purchase, he is willing to spend 200 Yuan to buy it. After watching, he is shocked by the magnificent scene of Liu Sanjie, attracted by the visual effects of light and water, and immersed in costumes and Folk Songs full of ethnic characteristics. He will be satisfied, and the marginal utility obtained is 400 utility units. At this time, he can obtain the maximum marginal utility. Thanks to the good impression left on the first time, he is willing to spend 160 Yuan to buy it, making the second purchase. Since he already knows all the content, his marginal utility is 320 utility units, which is a decline compared with the previous one. Until the tourists buy the impression Liu Sanjie for the sixth time, at the time, the marginal utility of buying the tourism product will be 0 utility units, and the price he is willing to pay is $¥ 0$ Yuan. It means that tourists are unwilling to buy this tourism product and will not buy the tourism product in the future.

\section{Analysis of the causes of the diminishing marginal utility of "Impression Liu Sanjie" cultural tourism products}

According to the previous calculation and analysis, the marginal utility of tourists buying "Impression Liu Sanjie" cultural tourism products shows a decreasing trend, which is a common problem in all tourism products, especially cultural tourism products. The main reason is the serious homogeneity of products, the lack of distinctiveness, innovation and in-depth product cultural exploration, the lack of strong brand positioning and the gap between highend consumption, etc.

\subsection{Intensified competition among peripheral homogenized products, and the characteristic advantages of products have gradually declined}

"Impression Liu Sanjie", the 2003 premiere shocked China and foreign countries, and the benefits were impressive. However, with the advent of impression series such as Impression Lijiang in 2006, Impression West Lake in 2007, Impression Dahongpao in 2010 and Impression Wulong in 2011, the number of homogenized products is increasing. The advantages of "Impression Liu Sanjie" no longer exist, competition intensifies, and product characteristics gradually decline. According to the law of diminishing marginal utility, when a tourist purchases a certain type of cultural tourism product for the first time, he will be satisfied and obtain the greatest marginal utility. However, when he buys the same type of products for many times, he will find that these products are all the same, without forming their own characteristics, their marginal utility will be reduced, and he will not buy this type of tourism products. 


\subsection{The products lack innovation and the return rate of tourists is low}

On the one hand, "Impression Liu Sanjie" were combined with the local cultural characteristics when developing cultural tourism, but did not delve into the local cultural connotations. On the other hand, the cultural tourism product of "Impression Liu Sanjie" has remained unchanged for more than ten years after its success. It has not innovated on the original basis and kept pace with the times. From the perspective of the law of diminishing marginal utility, after the first purchase, tourists will not make second purchase in order to maintain the maximum marginal benefit without innovation. Therefore, the cultural tourism products of "Impression Liu Sanjie" cannot escape the result of being abandoned by tourists.

\subsection{The overall scale is small and lacks a strong brand}

The scale of cultural tourism development is small. The "Impression Liu Sanjie" scenic spot is small in scale. There are no other cultural tourism products in the scenic spot, or even other tourist products that can be visited and played. In addition, the nearest cultural tourism product around "Impression Liu Sanjie" is Yangshuo West Street, which is about 2 kilometers away, and there are no other cultural tourism products. It lacks competitiveness and has not formed its own brand advantage. The profit is small, and the tourists' sense of experience is also relatively poor. They cannot meet their consumption needs, and they cannot keep the marginal utility greater than zero.

\subsection{Positioning is not accurate enough, and there is a gap with high-end oriented}

Due to the limited vision of the managers of cultural tourist attractions, insufficient understanding of the culture in the area, or short-term interest considerations, they have insufficient knowledge of cultural tourist attractions and cannot accurately judge the advantages and disadvantages of their development. This leds to inaccurate positioning of "Impression Liu Sanjie" during development. There is a gap between its existing products and the development trend of high-end tourism, which cannot meet the needs of tourists for in-depth experience and research tourism. Therefore, the cultural tourism products of "Impression Liu Sanjie" were not very attractive to tourists from the beginning, so his initial marginal utility was relatively low.

\section{Suggestions on improving the marginal utility of "Impression Liu Sanjie" cultural tourism products}

\subsection{Dig deeper into local culture and create local characteristic products}

"Impression Liu Sanjie" was able to achieve great success at the beginning. One of the reasons is that Impression Liu
Sanjie integrates Guilin's national culture with Guilin's unique landscape. This is something other scenic spots cannot imitate, let alone replace it and become Guilin's main product to attract tourists. Therefore, the scenic spot should dig into the local culture of Yangshuo on the basis of this advantage, form its own unique cultural tourism characteristics, and create local characteristic tourism products. In this way, the cultural tourism products of "Impression Liu Sanjie" can form a greater attraction to tourists and maximize the initial marginal utility.

\subsection{Continue to innovate tourism products and maintain a positive marginal utility}

According to Figure 3 Tourism product life cycle curve, when cultural tourism products enter a mature period, tourist attractions will improve cultural tourism products, such as improving product quality, increasing product functions, changing product forms, and adding new elements. Improvements from a small aspect can stimulate a new hot spot of tourism demand and increase the revisit rate of tourists. Therefore, the law of marginal decline can always be greater than zero and remain positive. Therefore, "Impression Liu Sanjie" asked that tourism products should keep pace with the times, and constantly inject new elements according to the needs of tourists to make it attractive to tourists.

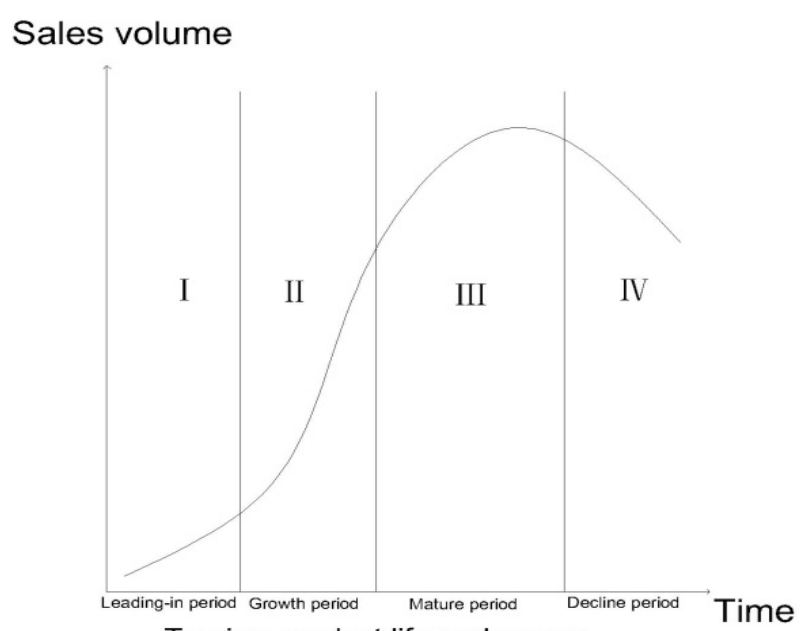

Tourism product life cycle curve

Figure 3 Tourism product life cycle curve

\subsection{Expand the scale of cultural tourism development and establish a strong local brand}

"Impression Liu Sanjie" should continuously add new elements according to the local cultural characteristics and the needs of tourists, expand the scale of cultural tourism development, provide tourists with more choices, enhance the experience of tourists, and extend the cycle of the law of diminishing marginal utility. "Impression Liu Sanjie" and its surrounding cultural tourism products should focus on the local characteristic culture, creating a unique local cultural brand. And through various publicity methods, the brand's popularity has been promoted, making it a major factor in attracting tourists to Yangshuo, driving the development of other tourism products and the economic development of the surrounding area. 


\subsection{Clarify your own advantages and disadvantages, and seek high-end tourism positioning}

For all cultural tourist attractions, their service targets are tourists. Therefore, the "Impression Liu Sanjie" scenic spot should clarify its own problems and disadvantages, identify its own positioning, and constantly seek high-end tourism positioning, based on the strong demand of tourists for in-depth experience and research tourism and the cultural connotation of Yangshuo. In particular, it is necessary to strengthen the service capabilities for highend tourism, cultivate high-end tourism talents, improve the experience satisfaction of tourists, and let them have a memorable experience, so that the marginal utility remains always positive.

\section{Conclusions}

From the perspective of economics, this paper studies tourists' marginal utility of cultural tourism products of "Impression Liu Sanjie", and the results show that the marginal utility of tourists buying "Impression Liu Sanjie" cultural tourism products shows an obvious decreasing trend. These are common problems of these tourism products, especially cultural tourism products. "Impression Liu Sanjie" needs to dig deeper into local culture, continuously innovate tourism products, establish strong local brands, and seek high-end tourism positioning in order to remain invincible in the future competition. In the context of cultural and tourism integration, this article provides a technical reference for solving the current problems of "Impression Liu Sanjie", such as the decline in competitiveness and low operating efficiency. And it is conducive to the sustainable development of "Impression Liu Sanjie" cultural tourism products.

\section{Acknowledgements}

This article is one of the phased results of the key support project of the Ministry of Culture and Tourism for graduate students "Research on the Integrated Development Path of Guangxi Zhuang Nationality's Traditional Sports Culture and Tourism from the Perspective of Cultural Experience" (WLRCY2019-070) and the Guangxi Postgraduate Innovation Plan Project "Analysis of the Temporal and Spatial Patterns of Guangxi Tourism Development and Optimization Strategies" (YCSW2019164).

\section{Reference}

1. Qin, J., Zhang, J. J. (2020), The origin and development of local tourism culture in Guanyangwen. J. Tourism Today, 18:20-21.

2. Song, F.Y. (2020), The influence of Chinese traditional culture on tourism management. J. Tourism Today, 18:41-42.

3. Xia, F., Wang, J. J. (2020), Optimized design of cultural tourism products in Linli county. J. Tourism
Today, 18:12-14.

4. Luo, Y. M. (2020), A probe into the development and promotion of ethnic cultural tourism in Qiannan prefecture. J. Tourism Today, 18:15-17.

5. Bai, C. (2020), Development Strategy of Inner Mongolia Folk Culture Tourism. J. Tourism Today, 18:54-55

6. Gao, H. Y. (2007) Utility theory. Gao, H. Y.(Eds.), Western Economics (Micro Part) Fifth Edition. China Renmin University Press, Beijing. pp. 57-97.

7. Zhang, X. Y. (2018), . The Development of Guizhou Tourism Based on the Law of Diminishing Marginal Utility. J. ECONOMIC RESEARCH GUIDE, $18: 157-158,172$.

8. Xiang, M. H.(2019), Research on the Development of Tibet's Tourism Industry under the Law of Diminishing Marginal Utility. J. Operators, 33:5-6.

9. Sun, Y. K., Liao, M.C. (2011), Influence of Law of Diminishing Marginal Utility on Tourism Products and its Precautions. J. Journal of Suihua University, 31:62-64. 